\title{
Clinical and Diagnostic Value of Highly Sensitive Cardiac Troponins in Arterial Hypertension
}

\author{
Aleksey Chaulin $\mathbb{1 D}^{1,2}$ \\ 'Department of Cardiology and \\ Cardiovascular Surgery, Samara State \\ Medical University, Samara, 443099 , \\ Russia; ${ }^{2}$ Department of Histology and \\ Embryology, Samara State Medical \\ University, Samara, 443099, Russia
}

\begin{abstract}
In modern laboratory diagnostics of cardiovascular diseases (CVD), there is a clear tendency toward an increase in the sensitivity of methods for determining key CVD biomarkers, among which highly sensitive cardiac troponins (hs-Tn) deserve special attention. The introduction of the latter into clinical practice made it possible not only to improve the early diagnosis of acute myocardial infarction but also to open up a number of additional valuable opportunities for the use of hs-Tn, including the assessment of the risk of developing CVD in a healthy population, detection and monitoring of early myocardial injuries in the early stages of CVD development (for example, with ischemic heart disease and arterial hypertension), with noncardiac pathologies (for example, sepsis, chronic obstructive pulmonary disease, chronic renal failure, stroke, cancer, etc), and diagnostics of CVD by using biological fluids that can be obtained by noninvasive methods. This article discusses in detail the diagnostic value of hs-Tn in serum and urine in cases of arterial hypertension. Also, the paper pays considerable attention to the consideration of the mechanisms underlying the increase in hs-Tn in serum and urine in cases of arterial hypertension.
\end{abstract}

Keywords: CVD, hs-Tn, acute myocardial infarction, arterial hypertension, hypertensive crisis, noninvasive diagnostics, oral fluid, urine

\section{Introduction}

Troponin-tropomyosin complex (TTC) localized on thin filaments in cardiomyocytes of myocardium tissue and on myosymplasts of skeletal muscle tissue is the most critical regulator of contractile function. ${ }^{1,2}$ TTC includes four proteins (troponin T, troponin C, troponin I and tropomyosin), each of them has specific functions in the process of striated muscle contraction: troponin $\mathrm{T}$, a tropomyosin-binding subunit, fixes the troponin complex on thin actin filaments; troponin $\mathrm{C}$, a calcium-binding subunit, binds calcium ions entering the cytoplasm from the sarcoplasmic reticulum in contraction stimulation; troponin I, an inhibitory subunit, blocks adenosine triphosphate hydrolysis and prevents interaction of actin with myosin in the absence of calcium ions during muscle relaxation. ${ }^{3,4}$ The importance of these proteins in the regulation of myocardium contractile function is confirmed by the fact that even minor mutations leading to the change in only one or several amino acids in TTC proteins often cause contractile function disorder and development of hereditary cardiomyopathies. ${ }^{4-6}$ The amino acid composition of two TTC components (troponin T and troponin I) in myocardium and skeletal muscle tissues is different, while the amino acid structure of troponin $\mathrm{C}$ and tropomyosin is absolutely identical and does not allow using the latter as biomarkers of myocardial injury. ${ }^{7-9}$
Correspondence: Aleksey Chaulin Department of Cardiology and Cardiovascular Surgery, Department of Histology and Embryology Samara State Medical University, 89 Chapaevskaya Street, Samara Region, Samara, 443099, Russia Tel + 7927 770-25-87

Email alekseymichailovich22976@gmail.com 
Clinical and diagnostic value of serum cardiac troponin (cTnT and cTnI) levels depends principally on the method of their determination and differs in previously used (low sensitive and moderately sensitive) and modern (high sensitive and ultrasensitive, hs-cTnT and hs-cTnI) immunoassays for $\mathrm{cTnT}$ and $\mathrm{cTnI}$ determination rather significantly. ${ }^{10,11}$ Methods for cTnT and cTnI determination have been continuously developing since their creation in the late 1990s by two independent research groups led by Cummins et al and Katus et al, respectively. ${ }^{12-14}$ While methods for cTnT and cTnI determination have developed, significant changes in our understanding of the biology and clinical and diagnostic value of these biomarkers have occurred. ${ }^{10,14,15}$ For example, firstand second-generation immunoassays conventionally referred to as low sensitive could detect only large focal acute myocardial infarctions, and the time of detection diagnostically significant $\mathrm{cTnT}$ and $\mathrm{cTnI}$ levels from the moment of chest pain onset was very significant (on average 12-24 h). Moreover these methods had a significant disadvantage in the form of cross (nonspecific) reactions of diagnostic antibodies (anti-cTnT and anti-cTnI) with skeletal troponin molecules that led to false positive results in some patients with skeletal muscle injuries (in different hereditary and acquired musculoskeletal myopathies) ${ }^{16,17}$ or after extreme physical stress. ${ }^{18,19}$ Perhaps this circumstance is essential in the developing of likely erroneous hypotheses regarding the extracardiac expression of cardiac troponins in skeletal muscles. ${ }^{20-24}$ Thus, Austrian researchers Schmid et al did not confirm data on extracardiac cTnT and cTnI expression in skeletal muscle samples obtained from patients with hereditary and acquired musculoskeletal myopathies in the recent study. ${ }^{25}$ Although many previous research groups including Apple et $\mathrm{al}^{20}{ }^{20}$ Ricchiutti et $\mathrm{al}^{21}{ }^{21}$ Messner et $\mathrm{al}^{22}$ McLaurin et $\mathrm{al}^{23}$ and Jaffe et $\mathrm{al}^{24}$ consistently noted extracardiac expression in human striated skeletal muscles and the possibility of increased cTnT and cTnI levels due to damage of exactly skeletal muscles but not of myocardium. Although the recent study of Schmid et al did not confirm the hypothesis of possible extracardiac cTnT and cTnI expression, they identified a rather important problem included cross-reactivity of modern anti-cTnT and anticTnI antibodies (which are key components of highly sensitive immunoassays) with skeletal troponins that led to false positive increase of hs-cTnT and hs-cTnI levels in patients without cardiovascular diseases (CVD). It is quite remarkable that cross-reactivity is significantly more specific for hs-cTnT (levels are increased in almost 70\% of patients with musculoskeletal myopathies) and less specific for hs-cTnI (levels are increased only in $4 \%$ of patients with musculoskeletal myopathies). ${ }^{25}$ Thus, the problem of cross (nonspecific) reactivity remained even in the most modern troponin immunoassays that indicates the need for additional research to specify the causes of this phenomenon and its correction. At the same time, more detailed attention should also be paid to the design and results of the study conducted by Schmid et $\mathrm{al}^{25}$ according to which most of the examined patients with elevated hs-cTnT level had hereditary myopathies which are characterized by genetic mutations in the composition of proteins, sarcomere including TTC components. Mutations (by the type of amino acid substitution or deletion) in protein molecules that affect antigenic epitopes can lead to the change both in the functioning of protein molecules and in the change of antigen-antibody interaction which is a key step in the immunoassay. ${ }^{13}$ It can be assumed that some of the false positive reactions may be due to a similar phenomenon.

Although the absolute cardiac specificity of cardiac troponins is beyond doubt in most researchers, there are many reasons and mechanisms for increased cardiac troponins levels in noncardiac and systemic conditions, both physiological and pathological ones. On the one hand this circumstance can complicate the differential diagnosis of AMI from these conditions, but on the other hand, it opens up additional diagnostic possibilities for use in practical medicine. ${ }^{9,26-28}$ Currently all reasons of increased cardiac troponins that are not associated with AMI can be conditionally divided into three groups (Figure 1). And it should be noted that just the highly sensitive methods for troponin determination revealed a significant part of these reasons of increased cTnT and cTnI.

Our understanding of troponin biochemistry/biology has changed significantly with the improvement of troponin immunoassays. For example, before the appearance of highly sensitive immunoassays, cTnT and cTnI were considered strictly intracellular molecules whose detection in blood serum was considered as a key diagnostic criterion for AMI. ${ }^{7-9}$ With the appearance of the first highly sensitive immunoassays in 2007-2010, cardiac troponins began to be detected in a significant number (more than 50\%) of healthy individuals examined (using highly sensitive first generation immunoassays). The subsequent improvement of highly sensitive immunoassays led to even more significant increases in sensitivity and these immunoassays 


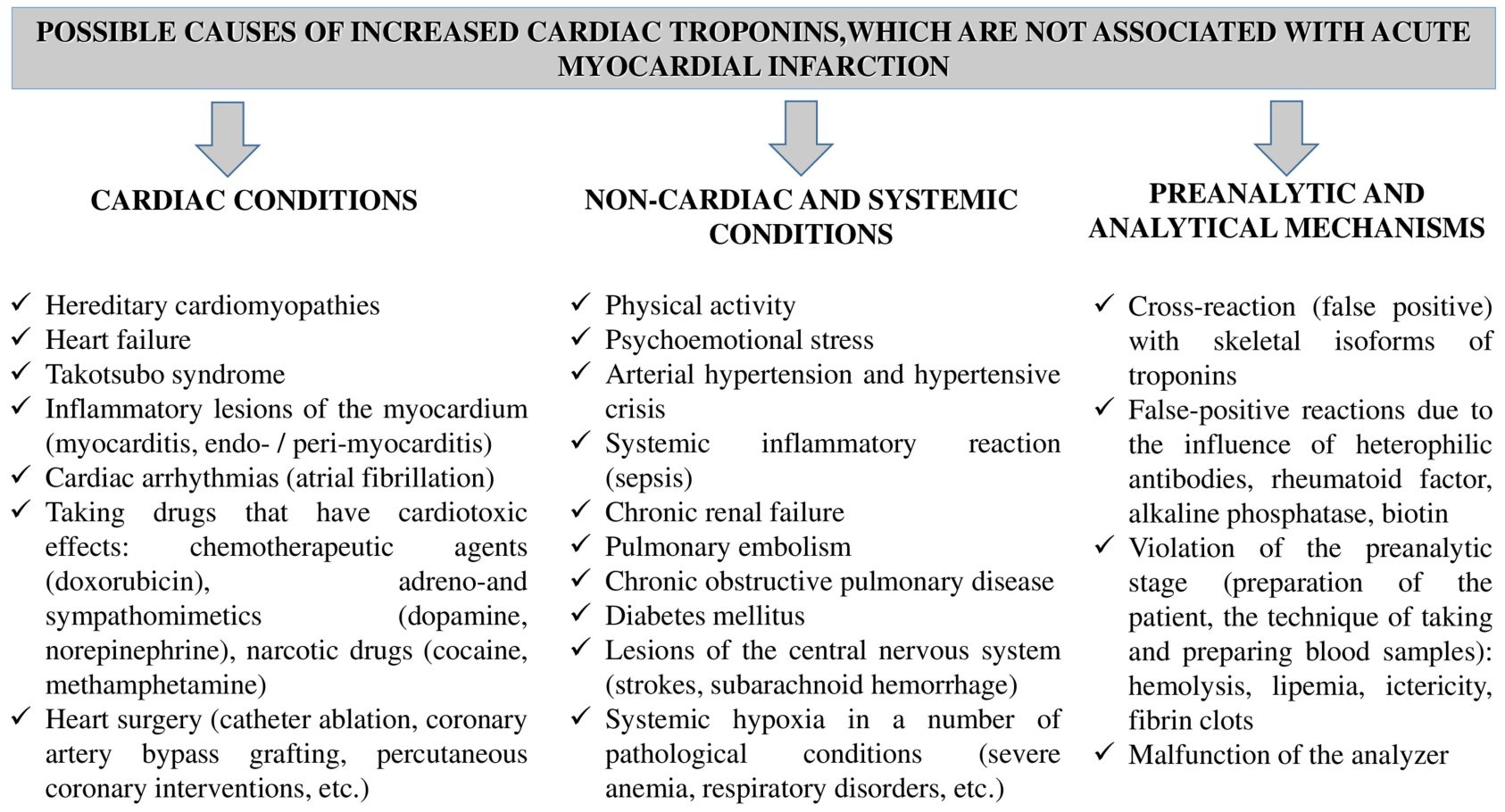

Figure I The main reasons of increased blood serum cardiac troponins which may not be associated with AMI. ${ }^{9,26-29}$

were designated as highly sensitive second, third generation immunoassays and, finally, the most modern immunoassays are also called ultrasensitive immunoassays (Table 1). The latest methods are ways to detect practically single molecules in the blood serum of all examined completely healthy patients that allows consideration cTnT and cTnI molecules as products of normal metabolism of

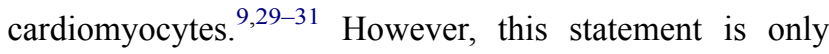
true if serum hs-cTnT and/or hs-cTnI levels are less than the 99th percentile (upper limit of normal) whereas above the 99th percentile, hs-cTnT and hs-cTnI levels may indicate the presence of any acute or chronic pathological process involving myocardial tissue. Highly sensitive immunoassays also revealed the dependence of hs-cTnT and hs-cTnI levels on the gender and age characteristics of patients that are proposed to be taken into account in modern algorithms for AMI diagnosis. ${ }^{9,32-34}$ The information on the existence of circadian rhythms for hs-cTnT and hs-cTnI according to which morning concentrations are higher than evening ones ${ }^{35-37}$ that some researchers consider to be due to increased activity of the sympathicoadrenal, hypothalamic-pituitary-thyroid and coagulation systems in the morning ${ }^{36}$ which is an evolutionally developed adaptive mechanism for the waking period is quite remarkable. ${ }^{37,38}$ At the same time, cases have been described according to which circadian fluctuations in hs-
cTnT levels can even affect AMI diagnosis. ${ }^{39,40}$ In the study design analysis by van der Linden et al, it was suggested that the degree of circadian fluctuations in hscTnT and hs-cTnI may be affected by concomitant (comorbid) pathologies, in particular, chronic renal failure (CRF) ${ }^{39}$ Taking into account the high prevalence of patients with comorbid pathologies, ${ }^{41}$ the significance of hs-cTnT and hs-cTnI circadian rhythms effect on AMI diagnosis can be very significant. Thus, further larger studies are required to establish the potential exact effect of hs-cTnT and hs-cTnI circadian rhythms on AMI diagnosis and the subsequent reflection of these features in modern diagnostic algorithms, if confirmed.

It should be also noted that due to modern methods for hs-cTnT and hs-cTnI determination new diagnostic possibilities for the use of these biomarkers in practical medicine have opened up. In particular, it has been demonstrated that hs-cTnT and hs-cTnI levels can be used to assess the risk of CVD (AMI, stroke, heart failure) (Table 2), ${ }^{42,43}$ to establish the prognosis for patients with both heart disease (myocarditis, endocarditis, cardiomyopathies) ${ }^{44-46}$ and noncardiac nosologies (chronic renal failure, sepsis, pulmonary embolism, chronic obstructive pulmonary disease, stroke, oncological diseases and others), ${ }^{26-28,47,48}$ identification of latent forms of coronary heart disease (CHD) using stress tests (physical or pharmacological stress) with subsequent 
Table I Classification of Troponin Immunoassays According to Their Sensitivity

\begin{tabular}{|l|l|l|}
\hline $\begin{array}{l}\text { Classification of Immunoassays According to Their } \\
\text { Sensitivity }\end{array}$ & $\begin{array}{l}\text { Lower Detection Limit } \\
\text { (Minimum Detectable } \\
\text { Concentration) of } \\
\text { Cardiac Troponins, ng/L }\end{array}$ & $\begin{array}{l}\text { The Number of Examined Healthy } \\
\text { Patients in Whom Cardiac Troponins } \\
\text { are Determined (Above 0 ng/L but } \\
\text { Not More Than 99th Percentile), \% }\end{array}$ \\
\hline Low sensitive immunoassays & $500-1000 \mathrm{ng} / \mathrm{L}$ & $0 \%$ \\
\hline Moderately sensitive immunoassays & $50-100 \mathrm{ng} / \mathrm{L}$ & Less than 50\% \\
\hline Highly sensitive Ist generation immunoassays & Dozens ng/L & $50-75 \%$ \\
\hline Highly sensitive 2nd generation immunoassays & Dozens ng/L & $75-95 \%$ \\
\hline Highly sensitive 3rd generation immunoassays & Several ng/L & More than $95 \%$ \\
\hline Highly sensitive 4th generation immunoassays (ultrasensitive) & Less than I ng/L & $99-100 \%$ \\
\hline
\end{tabular}

Table 2 Levels of hs-cTnI for Stratification of CVD Risk in the General Population ${ }^{43}$

\begin{tabular}{|l|c|c|}
\hline $\begin{array}{l}\text { Cardiovascular } \\
\text { Risk }\end{array}$ & $\begin{array}{c}\text { The Concentration } \\
\text { of hs-cTnl in the } \\
\text { Serum of Men }\end{array}$ & $\begin{array}{c}\text { The Concentration } \\
\text { of hs-cTnl in the } \\
\text { Serum of Women }\end{array}$ \\
\hline Low & $<4 \mathrm{ng} / \mathrm{L}$ & $<6 \mathrm{ng} / \mathrm{L}$ \\
Moderate & $4-10 \mathrm{ng} / \mathrm{L}$ & $6-12 \mathrm{ng} / \mathrm{L}$ \\
High & $>10 \mathrm{ng} / \mathrm{L}$ & $>12 \mathrm{ng} / \mathrm{L}$ \\
\hline
\end{tabular}

assessment of troponin levels, ${ }^{49}$ monitoring the degree of myocardial damage during cardiac toxic antitumor chemotherapy ${ }^{50,51}$ as well as in sports medicine/cardiology. ${ }^{52}$

One of the significant reasons for increased cardiac troponins deserving special attention is arterial hypertension (AH). In an extremely abundant number of review articles devoted to discussion of the prognostic value of hs-cTnT and hs-cTnI in many of the above cardiac and noncardiac conditions, insufficient attention is paid to such an important pathology as AH. It should be noted that there are currently no separate review articles devoted to detailed discussion of the diagnostic/prognostic significance of hs-cTnT and hs-cTnI and potential mechanisms of cardiac troponin molecule release from the myocardium in hypertension.

The aim of this review is to consider clinical and diagnostic value of hs-cTnT and hs-cTnI in human body fluids in $\mathrm{AH}$ as well as the potential mechanisms on which the increase in hs-cTnT and hs-cTnI concentration in hypertension is based.

According to modern data, hypertension has a huge impact on the risk of cardiovascular morbidity, mortality, and quality of life of patients worldwide. ${ }^{53,54}$ It was found that $\mathrm{AH}$ is the cause of occurrence of approximately $54 \%$ of stroke cases and $47 \%$ of CHD cases in patients annually. ${ }^{55}$ In 2013, the World Health Organization (WHO) recognized $\mathrm{AH}$ as the leading risk factor associated with mortality worldwide. ${ }^{56}$ At the same time, the prevalence of $\mathrm{AH}$ in the general population may be much higher than is currently believed, and many patients with high blood pressure (BP) may often be unaware about it and not receive appropriate treatment that raises particular fears among practitioners and researchers. In some cases, blood pressure levels can reach very high numbers, and patients may not even know about it. For example, in the study conducted by Caligiuri et al, they found that approximately $50 \%$ of people from the general population have elevated blood pressure while in $2 \%$ of them blood pressure levels were higher than $180 / 110 \mathrm{mmHg}$ and at the same time they did not receive antihypertensive drugs because they did not know about their hypertensive status. $^{53}$

\section{Cardiac Troponins in Arterial Hypertension: Clinical Study Data}

As noted above, the reasons and mechanisms for increased hs-cTnT and hs-cTnI are extremely variable and in most cases they do not always indicate the presence of AMI. Thus, in a large retrospective study conducted by Lindner et al, the main reasons for increased hs-cTnT in patients $(n=1573)$ who were admitted to the emergency department with complaints of chest pain and suspected AMI were analyzed. However, only in slightly more than 10\% (175 patients) of them AMI was subsequently diagnosed, and 1389 admitted patients had other causes of increased hscTnT not related to AMI. The analysis of reasons for the 
increase showed that the most significant included the following: chronic renal failure, cerebral ischemia, heart failure, pulmonary embolism, myocarditis, AH. And in $30 \%$ of cases, the authors failed to establish the cause of increased hs-cTnT. ${ }^{57}$ It can be assumed that some of these cases were due to false positive results and subclinical myocardial damage in a number of other nosologies. In recent analyses performed by Long et $\mathrm{al}^{58}$ and Masri et $\mathrm{al}^{59}$ $\mathrm{AH}$ is also mentioned as the significant cause of increased cardiac troponins not related to AMI although potential mechanisms of hs-cTnT and hs-cTnI increase in $\mathrm{AH}$ are not discussed.

Several studies have shown that in patients without CVD, hs-cTnT and hs-cTnI levels precede the development of $\mathrm{AH}$ and structural changes in the heart associated with $\mathrm{AH}{ }^{60-63}$ Thus, even early prehypertensive conditions can cause early structural (subclinical) changes in the heart such as left ventricle hypertrophy ( $\mathrm{LVH}$ ) providing potential opportunities for initiating preventive measures in revealing LVH. $^{60}$ Chronic subclinical myocardial damage which is well detected by modern highly sensitive troponin immunoassays are also early risk factors for AH development in the general population. A large study conducted by McEvoy et al included 6516 healthy subjects without signs of CVD, baseline concentrations were associated with the risk of $\mathrm{AH}$ and $\mathrm{LVH}$ during a six-year followup period. ${ }^{61}$ This study emphasizes the possibility of using hs-cTnT levels as laboratory biomarkers for assessing the risk of AH development which will identify those patients who should undergo a set of therapeutic and prophylactic measures to prevent $\mathrm{AH}$ and further dangerous cardiovascular complications following $\mathrm{AH}$. According to the study conducted by çar et al hs-cTnT levels in patients with $\mathrm{AH}$ and prehypertensive status are associated with $\mathrm{LVH}$ and worsening of geometric echocardiographic parameters of the left ventricle including left ventricular mass index and left ventricle wall thickness. ${ }^{63}$

According to some studies, AH can directly damage cardiomyocytes and cause an increase in cardiac troponin levels determined by highly sensitive methods. Thus, Harvell et al, noted that $\mathrm{AH}$ is the fourth leading cause of hs-cTnI increase after such conditions as AMI, infectious diseases and chronic renal failure. ${ }^{64}$ In a retrospective study conducted by Afonso et al which included 576 patients, it was found that hs-cTnI level is increased in $32 \%$ of patients with $\mathrm{AH}$. Average peak serum concentrations of cardiac troponin I were 4.06 $\pm 14.6 \mathrm{ng} / \mathrm{mL}$. The authors also found that patients with
$\mathrm{AH}$ and increased hs-cTnI levels had a lower left ventricular ejection fraction, increased heart rate and a higher prevalence of diabetes mellitus compared to the patients with $\mathrm{AH}$ and with normal hs-cTnI levels. ${ }^{65}$

According to the literature, approximately $1-2 \%$ of patients will develop $\mathrm{AH}$ hypertensive crisis (HC) during their lifetime. ${ }^{66}$ At $\mathrm{HC}$, the levels of systolic and diastolic blood pressure exceed 180 and $120 \mathrm{mmHg}$ correspondingly creating a high threat to the life of patients. It has been shown that without drug treatment, the mortality rate in $\mathrm{HA}$ is $70 \%$ and $90 \%$ within one year and five years, respectively. ${ }^{67}$ Moreover, among all target organs, the most frequently affected tissue is the myocardium which can be detected using highly sensitive methods for determination of cardiac troponins. Thus, in the recent study Acosta et al found that $15 \%$ of patients with HA had an increase in hs-cTnT concentration above diagnostic threshold (99th percentile) for AMI (>15 ng/L). ${ }^{68}$ Increased cardiac troponins in HA predict long-term adverse cardiovascular outcomes. So, according to Pattanshetty et al major adverse cardiac or cerebrovascular events (MACCE) (AMI, unstable angina, HC, pulmonary edema, stroke) developed during the two-year follow-up period much more often in those patients with $\mathrm{HC}$ who had increased hs-cTnI level (71\%) compared to patients with hs-cTnI level within the normal range $(38 \%)$. In addition, patients with increased hs-cTnI levels were also more likely to have obstructive CHD (odds ratio (OR) 8.97; 95\% confidence interval $(\mathrm{CI}):=1.4-55.9 ; P<0.01) .{ }^{69}$ In another retrospective cohort study, which included 929 patients with $\mathrm{HC}$, an increased hs-cTnI level was recorded in about one third of patients. At the same time, in patients with increased hs-cTnI levels such adverse events as myocardial infarction (66\% vs 34\%; OR: 4.74; 95\%CI: $2.74-$ $8.20 ; P<0.001)$ and pulmonary edema $(50 \%$ vs $30 \%$; OR: 2.31; 95\%CI: 1.24-4.30; $P=0.007$ ) developed more often. Even after adjusting for relevant covariates (gender, race, history of diabetes mellitus, heart failure, CRF, and CHD), the predictive value of hs-cTnI in detection remained significant. $^{70}$ According to another study conducted by Omondi et al, the prevalence of increased cTnT levels in patients with $\mathrm{HC}$ is $7 \%$. At the same time, during the average six-month and one-year follow-up periods mortality in the group of patients with increased cTnT level was $27 \%$ and $32 \%$, respectively, while in the group of patients with normal concentration of cTnT it was only $5 \%$ and $8 \%$, respectively. According to statistical analysis, patients with $\mathrm{HC}$ and increased cTnT have a higher risk of death 
within six months (OR: 6.4; 95\%CI: 2.1-19.2) and within one year (OR: 3.7 ; 95\%CI: 1.4-9.8) compared to patients with normal cTnT levels. ${ }^{71}$

Thus, cTnT and cTnI determined using modern high sensitive and ultrasensitive immunoassays allowed detection of minor and subclinical myocardial damage during prehypertensive status (before AH development), actually in AH and HC. At the same time, increased hs-cTnT and hs-cTnI levels have a high prognostic value allowed the identification of those patients with $\mathrm{AH}$ and $\mathrm{HC}$ who required a more thorough examination and the use of a complex of therapeutic and prophylactic measures to reduce the risk of developing dangerous adverse consequences and complications in future.

\section{Potential Mechanisms on Which Increased hs-cTnT and hs-cTnl Levels in Human Body Fluids are Based in AH}

In general, the following potential mechanisms of hs-cTnT and hs-cTnI increase in body fluids in $\mathrm{AH}$ and $\mathrm{HC}$ can be identified: (1) activation of the processes of programmed cell death (apoptosis) caused by excessive distension of myocardial tissue and increased activity of the adrenergic system; (2) activation of the processes of proteolytic degradation of cTnT and cTnI molecules by various enzymes in cardiomyocytes and in blood serum; (3) increased membrane permeability of cardiomyocytes due to myocardial overload and/or membrane protein-lipid components damage by proteases; (4) the effect of blood pressure on the processes of $\mathrm{cTnT}$ and $\mathrm{cTnI}$ elimination from blood serum by filtration in the kidneys and functional activity of the liver; (5) increased myocardial hypertrophy due to increased blood pressure and increased release of molecules from cardiomyocytes; (6) stress/tension of the left ventricle wall.

\section{Activation of the Processes of} Programmed Cell Death (Apoptosis) Caused by Excessive Distension of Myocardial Tissue and Increased Activity of the Adrenergic System

According to a number of experimental studies, $\mathrm{AH}$ and $\mathrm{HC}$ increase the load on the myocardium causing distension of its walls that can activate apoptosis of cardiac myocytes. Thus, in the experimental study Cheng et al demonstrated that distension of the myocardial walls is closely related to the activation of the programmed cell death processes through increased oxidative stress (generation of reactive oxygen and nitrogen species) and increased expression of the apoptosis regulator-Fas protein. $^{72}$ Another mechanism that can enhance the processes of cardiomyocyte apoptosis in $\mathrm{AH}$ and $\mathrm{HC}$ is excessive activation of the adrenergic system. Several experimental in vitro studies on isolated cultured cardiomyocytes have shown that stimulation of beta-adrenergic receptors with norepinephrone $\mathrm{e}^{73,74}$ and isoproterenol ${ }^{75}$ initiates apoptotic signals in cardiomyocytes by cAMP-dependent and NF2dependent mechanisms, respectively. ${ }^{73-75}$ Apoptosis of cardiomyocytes can lead to significant increase of cTnT and cTnI levels even in the absence of necrotic processes. The experimental work showed that a short-term overload of the left ventricle with pressure simulated by intravenous administration of phenylephrine to laboratory pigs caused an increase in apoptotic processes but there were no signs of necrosis. It is noteworthy that hs-cTnT level already exceeded the upper limit of the 99th percentile $30 \mathrm{~min}$ after phenylephrine administration, and after one hour troponin $\mathrm{T}$ concentration was very high $(856 \pm 956 \mathrm{ng} / \mathrm{L})$ and further continued to increase somewhat more slowly reaching $1.462 \pm 1.691 \mathrm{ng} / \mathrm{L} 24 \mathrm{~h}$ after start of the experiment. ${ }^{76}$ It is important to note that the strong dynamics of increased hs-cTnT levels in the first hour in this experiment differs from the dynamics of increased serum troponin levels in AMI. This is due to the fact that in the absence of the coronary bed block cTnT molecules enter the general circulation faster in contrast to persistent occlusion of the coronary vessels, which is specific to AMI in the first hour. A strong rise of cardiac markers including troponin $\mathrm{T}$ is possible in the case of coronary vessel recanalization ("washout" phenomenon).

\section{Activation of the Processes of Proteolytic Degradation of cTnT and cTnl Molecules by Various Enzymes in Cardiomyocytes and in Blood Serum}

One of the potential mechanisms of cTnT and $\mathrm{cTnI}$ molecules release in $\mathrm{AH}$ is the process of proteolytic degradation that causes fragmentation of cTnT and cTnI molecules into such small fragments that presumably they can pass through an intact cell membrane. Thus, it has been shown that mechanical distension of cardiomyocytes caused by pressure and/or volume overload triggers a cascade of intracellular signals leading to the activation of a number of intracellular proteinases including matrix metalloproteinase-2 (MMP-2) and 
matrix metalloproteinase-14 (MMP-14). ${ }^{77,78}$ These enzymes break down the $\mathrm{cTnI}$ molecule in cardiomyocytes into smaller fragments ${ }^{79,80}$ facilitating their release into the extracellular space through the integral cardiomyocyte membrane.

Moreover, in addition to MMP-2 and MMP-14, the enzyme calpain-1 may be responsible for the proteolytic cleavage of cTnI molecule. Experimental study on Langendorff isolated heart model demonstrated that an increase of myocardial preload activates calpain-mediated proteolysis of $\mathrm{cTnI}$ molecule independently from myocardial ischemia. Specific inhibition of enzyme calpain-1 and elimination of preload prevented proteolytic degradation of $\mathrm{cTnI} .{ }^{81,82}$

Hypothetically, the concentration of cardiac troponins in $\mathrm{AH}$ and $\mathrm{HC}$ may be changed due to the processes of extracellular cleavage of cTnT and cTnI molecules. In the recent study Russian biochemists Katrukha et $\mathrm{al}^{83}$ found that serum enzyme thrombin causes proteolytic degradation of the intact cTnT molecule into two fragments. Taking into account the fact that patients with $\mathrm{AH}$ and $\mathrm{HC}$ often have an increased activity of blood coagulation system enzymes including thrombin, ${ }^{84,85}$ we can talk about the potential influence of this mechanism on the proteolytic degradation of $\mathrm{cTnT}$ molecule. The processes of troponin cleavage in cardiomyocytes ${ }^{77-82}$ and in blood serum $^{83}$ described above will lead to changes in the composition and availability of some antigenic determinants of cTnT and cTnI molecules to which diagnostic anti-cTnT and anti-cTnI antibodies can be targeted that will change serum concentration of cardiac troponins. Moreover, the use of drugs that affect the activity of the enzyme thrombin, in particular anticoagulant agents, can also potentially affect cardiac troponin levels. Perhaps, this circumstance is one of the main factors causing differences in cTnT and cTnI concentrations which can be obtained at testing the same serum using different testing systems in which diagnostic anti-cTnT and anti-cTnI antibodies are targeted to different epitopes of cTnT and cTnI molecules. In addition, the fragmentation of full-size cTnT and cTnI molecules into smaller compounds can enhance their elimination into urine and oral fluid through the renal and blood salivary barriers, respectively. On the one hand more rapid fragmentation and elimination of troponins can reduce the diagnostic value of blood serum troponins in the use of some diagnostic testing systems, and on the other hand, this mechanism opens up new diagnostic possibilities for determination $\mathrm{cTnT}$ and $\mathrm{cTnI}$ in urine and oral fluid that will be discussed later in this paper.

\section{Increased Membrane Permeability of Cardiomyocytes Due to Myocardial Overload and/or Membrane Protein-Lipid Components Damage by Proteases}

Membrane permeability of cardiomyocytes is an important factor determining the potential of the release of small molecules localized in the cytoplasm of myocytes into extracellular fluid and blood serum. In addition to the structural pool of cardiac troponins (localized within the TTC and performing a regulatory function), there is a nonstructural (unbound) pool of troponins in the cytoplasm of cardiomyocytes. The nonstructural troponin pool contains on average $6-8 \% \mathrm{cTnT}$ and $3-8 \% \mathrm{cTnI}$ of the total mass of troponin proteins in the cell, and these molecules are not involved in myocardial contraction. ${ }^{86}$ According to current opinion, cTnT and cTnI molecules which are the part of the nonstructural pool of troponins can be freely released in small amounts in completely healthy patients providing a normal (less than 99th percentile) baseline blood serum concentration. At minor and reversible damage of cardiomyocytes which, for example, can be observed during psychoemotional stresses ${ }^{87}$ and prolonged/high sports activity, ${ }^{88}$ the degree of molecule release from the nonstructural pool of troponins can exceed the upper limit of the 99th percentile by three-to-five times.

Integrin proteins, transmembrane glycoprotein receptors that bind the extracellular matrix to the intracellular cytoskeleton, play an important role in the regulation of the membrane permeability of cardiomyocytes and regulation of proteolytic degradation of $\mathrm{cTnT}$ and $\mathrm{cTnI}$ molecules. Thus, Hessel et al found that myocardium overloading leads to its distension and subsequent activation of integrins functioning as mechanotransducers. ${ }^{89}$ The activation of integrins causes an increase of enzymes MMP-2 and calpain-1 activity leading to proteolytic cleavage of cTnI and an increase in the release of the nonstructural pool due to an increase of membrane permeability. ${ }^{89}$

\section{The Effect of Blood Pressure on the Processes of cTnTand cTnl Elimination from Blood Serum by Filtration in the Kidneys and Functional Activity of the Liver}

The functional state of kidneys plays an important role in the laboratory diagnostics of many diseases including CVD since kidneys are actually involved in the elimination of those biomarkers that are used in laboratory diagnostics. As a rule the decrease of the functional activity of renal filtration leads to 
increased concentration of laboratory biomarkers including cardiac troponins in patients without signs of CVD. ${ }^{90,91}$ Thus, the degree of $\mathrm{cTnT}$ and $\mathrm{cTnI}$ concentration increase depends both on the release from cardiomyocytes and on the mechanisms of elimination. Blood serum concentration of molecules can accumulate to extremely high values depending on the degree of glomerular filtration rate (GFR) decrease even in patients without signs of myocardial damage. ${ }^{92,93}$ Thus, it has been shown that lower GFR is associated with higher levels of hs-cTnT ${ }^{93}$ However, according to some studies, the possibility of cardiac troponin elimination through the renal filter was considered unlikely since $\mathrm{cTnT}$ and $\mathrm{cTnI}$ were detected in the urine of a small number of patients. ${ }^{94,95}$ However, in these studies, moderately sensitive methods for troponin determination the sensitivity of which, apparently, was insufficient for detection of cTnT and cTnI molecules in urine were used. With the development of immunoassays and the emergence of highly sensitive methods for cTnT and cTnI determination, our ideas about clinical and diagnostic value of troponins in urine changed and renal mechanism of troponin elimination was finally confirmed. In particular, in the study by Pervan et al, they found hs-cTnI in the morning urine portion of all patients. Interestingly, mean urine hs-cTnI levels were significantly higher in hypertensive patients than in the control group (26.59 $\mathrm{pg} / \mathrm{mL}$ vs $14.95 \mathrm{pg} / \mathrm{mL}, P=0.045) .{ }^{96}$ Based on these results, it can be assumed that at higher blood pressure and GFR, the elimination of $\mathrm{cTnT}$ and $\mathrm{cTnI}$ molecules by renal filtration is enhanced which can be used for diagnostic purposes.

Moreover, the recent experimental study on rats showed that, in addition to the kidneys, liver plays an important role in troponin elimination from blood serum. ${ }^{97}$ Thus, impaired liver function will also potentially contribute to increased serum cTnT and cTnI levels. Since $\mathrm{AH}$ and liver pathologies have many common risk factors and pathogenetic mechanisms, ${ }^{98}$ and these pathologies often occur in combination in many patients, impaired liver function will also contribute to increased serum hscTnT and hs-cTnI concentrations in $\mathrm{AH}$.

\section{Increased Myocardial Hypertrophy Due to Increased Blood Pressure and Increased Release of Molecules from Cardiomyocytes}

$\mathrm{AH}$ is accompanied by overload of the left ventricular myocardium and its gradual hypertrophy. Several studies showed that LVH correlates with hs-cTnT and hs-cTnI concentration $^{62,63,99}$ thereby indicating a higher level of cardiac troponin molecules release from hypertrophic cardiomyocytes. In addition, hs-cTnT and hs-cTnI levels are significantly higher in healthy men than in women ${ }^{100-102}$ that is also explained by higher myocardial mass. Gender characteristics in serum hs-cTnT and hs-cTnI levels are specific for all highly sensitive methods used. ${ }^{102}$ Along with hs-cTnT and hs-cTnI, gender characteristics are also specific for another muscular biomarker (creatine phosphokinase, CPK) ${ }^{103,104}$ finally confirming the fact that during normal myocyte metabolism more cTnT, cTnI and CPK molecules are released into the blood serum in men than in women.

\section{Stress/Tension of the Left Ventricle Wall}

AH can also cause myocardial overload and increase the left ventricular wall stress that can lead to decrease of blood supply to the myocardial subendocardial layers and death of cardiomyocytes which is accompanied by increased cardiac troponin levels and decreased left ventricular systolic function. ${ }^{105,106}$

\section{On New Possibilities for Study hs-cTnT and hs-cTnl Levels in Body Fluids in $\mathrm{AH}$ and $\mathrm{HC}$ Obtained by Noninvasive Methods}

In a number of CVDs including AMI, heart failure and AH it is possible to use human body fluids including oral fluid (saliva) and urine obtained by noninvasive methods as an alternative diagnostic approach. ${ }^{96,107-112}$ Several recent studies using modern immunoassays have shown that hs$\mathrm{cTnI}^{107,108}$ and brain natriuretic peptide ${ }^{110}$ levels in the saliva of patients with AMI and heart failure are higher than in the control group, respectively. These works prove the existence of the mechanism for elimination of these cardiac markers molecules through the blood-salivary barrier. Considering the fact that hs-cTnI level in the morning urine of patients with $\mathrm{AH}$ is significantly higher than in the urine of normotensive patients, and use of antihypertensive drugs leads to the normalization of urinary hs-cTnI concentrations, hs-cTnI measurement can be considered a new promising tool for diagnostics and monitoring of patients with $\mathrm{AH}$ and $\mathrm{HC} .{ }^{96}$ In the recent study, urinary troponin levels may be predictive in patients with diabetes mellitus. Thus, hs-cTnI concentrations in morning urine more than $4.1 \mathrm{pg} / \mathrm{mL}$ are associated with the risk of short-term adverse cardiovascular events. In outpatient setting, the 
use of this noninvasive approach is more convenient and safer than testing serum. ${ }^{111}$ It has been shown that testing of hs-cTnT and hs-cTnI concentration in urine can be used in pregnant women to detect preeclampsia and myocardial damage. At the same time, researchers propose the development of test strips for hs-cTnT and hs-cTnI detection in urine. $^{112}$

The specific mechanisms by which cTnT and cTnI molecules pass through the three-layer renal filter (barrier) or hematosalivary barrier are not completely known and are controversial. The intact cTnT and cTnI protein molecules are rather large compounds and therefore cannot easily pass through the structural components of the renal and hematosalivary barriers. However, cTnT and cTnI molecules are sensitive to the action of various proteases (calpain-1, MMP, thrombin, and others) which cause their fragmentation into many fragments of lower molecular weight. Most likely these small fragments can freely pass through the pores of renal and hematosalivary barriers. Further clarification of these mechanisms of cTnT and cTnI fragmentation and elimination as well as identification of factors that affect these mechanisms is an important task and the subject of future research aimed to increasing clinical and diagnostic value of hs-cTnT and hs-cTnI in human body fluids.

\section{Conclusion}

Thus, due to the improvement of methods for cardiac troponin determination, new opportunities for the use of hs-cTnT and hs-cTnI in practical medicine have opened up. According to the clinical studies reviewed, arterial hypertension can be considered to be one of the most significant reasons of increased hs-cTnT and hs-cTnI. Increased hscTnT and hs-cTnI levels have a high predictive value that allowed the identification of those patients with prehypertensive conditions, arterial hypertension and hypertensive crisis who have a high risk of adverse cardiovascular events in the short- and long-term. Based on the analysis of literature data, several mechanisms on which the increase of hscTnT and hs-cTnI in human body fluids are based in arterial hypertension: activation of apoptosis processes, increased membrane permeability of cardiomyocytes, increased processes of intra- and extracellular proteolysis of cardiac troponin molecules, the effect of blood pressure on the processes of cardiac troponin molecules elimination by renal filtration and liver function, increased myocardial hypertrophy and left ventricular wall stress. A very interesting direction of further studies is the clarification of the diagnostic value of hs-cTnT and hs-cTnI in body fluids obtained by noninvasive methods in arterial hypertension and a number of other cardiac and noncardiac pathological conditions that cause myocardial damage.

\section{Disclosure}

The author reports no conflicts of interest in this work.

\section{References}

1. Kobayashi T, Jin L, de Tombe PP. Cardiac thin filament regulation. Pflugers Arch. 2008;457(1):37-46. doi:10.1007/ s00424-008-0511-8

2. Tobacman LS. Thin filament-mediated regulation of cardiac contraction. Annu Rev Physiol. 1996;58:447-481. doi:10.1146/ annurev.ph.58.030196.002311

3. Chaulin AM, Karslyan LS, Duplyakov DV. Non-Coronarogenic Causes of Increased Cardiac Troponins in Clinical Practice. J Clin Practice. 2019;10(4):81-93. doi:10.17816/clinpract16309

4. Duplyakov DV, Chaulin AM. Mutations of heart troponines, associated with cardiomyopathies. Kardiologiya. 2019;7 (3):8-17. doi:10.24411/2309-1908-2019-13001

5. Yotti R, Seidman CE, Seidman JG. Advances in the Genetic Basis and Pathogenesis of Sarcomere Cardiomyopathies. Annu Rev Genomics Hum Genet. 2019;20:129-153. doi:10.1146/annurevgenom-083118-015306

6. Marques MA, de Oliveira GA. Cardiac Troponin and Tropomyosin: structural and Cellular Perspectives to Unveil the Hypertrophic Cardiomyopathy Phenotype. Front Physiol. 2016;7:429. doi:10.3389/fphys.2016.00429

7. Thygesen K, Alpert JS, Jaffe AS, et al. Executive Group on behalf of the Joint European Society of Cardiology (ESC)/ American College of Cardiology (ACC)/American Heart Association (AHA)/World Heart Federation (WHF) Task Force for the Universal Definition of Myocardial Infarction. Fourth Universal Definition of Myocardial Infarction (2018). Glob Heart. 2018;13(4):305-338. doi:10.1016/j.gheart.2018.08.004

8. Chaulin AM, Duplyakov DV. Biomarkers of acute myocardial infarction: diagnostic and prognostic value. Part 1. J Clin Practice. 2020;11(3):75-84. doi:10.17816/clinpract34284

9. Chaulin A. Cardiac Troponins: contemporary Biological Data and New Methods of Determination. Vasc Health Risk Manag. 2021;17:299-316. doi:10.2147/VHRM.S300002

10. Shah A, Griffiths M, Lee KK, et al. High sensitivity cardiac troponin and the under-diagnosis of myocardial infarction in women. BMJ. 2015;350:g7873. doi:10.1136/bmj.g7873

11. Chaulin AM, Karslyan LS, Bazyuk EV, et al. Clinical and diagnostic value of cardiac markers in human biological fluids. Kardiologiia. 2019;59(11):66-75. doi:10.18087/cardio.2019.11. n414 In Russian.

12. Katus HA, Looser S, Hallermayer K, et al. Development and in vitro characterization of a new immunoassay of cardiac troponin T. Clin Chem. 1992;38(3):386-393. doi:10.1093/clinchem/ 38.3.386

13. Cummins B, Auckland ML, Cummins P. Cardiac-specific troponin-I radioimmunoassay in the diagnosis of acute myocardial infarction. Am Heart J. 1987;113(6):1333-1344. doi:10.1016/ 0002-8703(87)90645-4

14. Kozinski M, Krintus M, Kubica J, Sypniewska G. Highsensitivity cardiac troponin assays: from improved analytical performance to enhanced risk stratification. Crit Rev Clin Lab Sci. 2017;54(3):143-172. doi:10.1080/10408363.2017.1285268 
15. Brinkmann C, Schofer J. Allgemeine Definition des Myokardinfarkts 2018: was ist neu? [4th universal definition of myocardial infarction 2018: what is new?]. Herz. 2018;43 (8):681-688. doi:10.1007/s00059-018-4763-6

16. Löfberg $M$, Tähtelä $R$, Härkönen $M$, Somer H. Myosin heavy-chain fragments and cardiac troponins in the serum in rhabdomyolysis. Diagnostic specificity of new biochemical markers. Arch Neurol. 1995;52(12):1210-1214. doi:10.1001/ archneur.1995.00540360090020

17. Müller-Bardorff M, Hallermayer K, Schröder A, et al. Improved troponin T ELISA specific for cardiac troponin $\mathrm{T}$ isoform: assay development and analytical and clinical validation. Clin Chem. 1997;43(3):458-466. doi:10.1093/clinchem/43.3.458

18. Siegel AJ, Lewandrowski KB, Strauss HW, Fischman AJ, Yasuda T. Normal post-race antimyosin myocardial scintigraphy in asymptomatic marathon runners with elevated serum creatine kinase $\mathrm{MB}$ isoenzyme and troponin $\mathrm{T}$ levels. Evidence against silent myocardial cell necrosis. Cardiology. 1995;86(6):451-456. doi: $10.1159 / 000176922$

19. Apple FS. Tissue specificity of cardiac troponin I, cardiac troponin T and creatine kinase-MB. Clin Chim Acta. 1999;284 (2):151-159. doi:10.1016/s0009-8981(99)00077-7

20. Apple FS, Ricchiuti V, Voss EM, Anderson PA, Ney A, Odland M. Expression of cardiac troponin T isoforms in skeletal muscle of renal disease patients will not cause false-positive serum results by the second generation cardiac troponin $\mathrm{T}$ assay. Eur Heart J. 1998;19(Suppl):N:N30-3.

21. Ricchiutti V, Apple FS. RNA expression of cardiac troponin $\mathrm{T}$ isoforms in diseased human skeletal muscle. Clin Chem. 1999;45(12):2129-2135. doi:10.1093/clinchem/45.12.2129

22. Messner B, Baum H, Fischer P, et al. Expression of messenger RNA of the cardiac isoforms of troponin T and I in myopathic skeletal muscle. Am J Clin Pathol. 2000;114(4):544-549. doi:10.1309/8KCL-UQRF-6EEL-36XK

23. McLaurin MD, Apple FS, Voss EM, Herzog CA, Sharkey SW. Cardiac troponin I, cardiac troponin T, and creatine kinase MB in dialysis patients without ischemic heart disease: evidence of cardiac troponin $\mathrm{T}$ expression in skeletal muscle. Clin Chem. 1997;43(6 Pt 1):976-982. doi:10.1093/clinchem/43.6.976

24. Jaffe AS, Vasile VC, Milone M, Saenger AK, Olson KN, Apple FS. Diseased skeletal muscle: a noncardiac source of increased circulating concentrations of cardiac troponin T. J Am Coll Cardiol. 2011;58 (17):1819-1824. doi:10.1016/j.jacc.2011.08.026

25. Schmid J, Liesinger L, Birner-Gruenberger R, et al. Elevated Cardiac Troponin T in Patients With Skeletal Myopathies. $J$ Am Coll Cardiol. 2018;71(14):1540-1549. doi:10.1016/j. jacc.2018.01.070

26. Chaulin AM, Duplyakov DV. Increased cardiac troponins, not associated with acute coronary syndrome. Part 1. Kardiologiya. 2019;7(2):13-23. doi:10.24411/2309-1908-2019-12002

27. Chaulin AM, Duplyakov DV. Increased cardiac troponins, not associated with acute coronary syndrome. Part 2. Kardiologiya. 2019;7(2):24-35. doi:10.24411/2309-1908-2019-12003

28. Chaulin AM, Svechkov NA, Volkova SL, Grigoreva YV. Diagnostic value of cardiac troponins in elderly patients without myocardial infarction. Modern problems sci educ. 2020;6. doi:10.17513/spno.30302

29. Chaulin AM, Karslyan LS, Grigorieva EV, Nurbaltaeva DA, Duplyakov DV. Metabolism of cardiac troponins (literature review). Complex Issues Cardiovascular Dis. 2019;8 (4):103-115. doi:10.17802/2306-1278-2019-8-4-103-115

30. Zhao H, Lin Q, Huang L, et al. Ultrasensitive chemiluminescence immunoassay with enhanced precision for the detection of $\mathrm{cTnI}$ amplified by acridinium ester-loaded microspheres and internally calibrated by magnetic fluorescent nanoparticles. Nanoscale. 2021;13(5):3275-3284. doi:10.1039/d0nr08008j
31. Garcia-Osuna A, Gaze D, Grau-Agramunt M, et al. Ultrasensitive quantification of cardiac troponin I by a single molecule counting method: analytical validation and biological features. Clin Chim Acta. 2018;486:224-231. doi:10.1016/j.cca.2018.08.015

32. Bohn MK, Higgins V, Kavsak P, Hoffman B, Adeli K. HighSensitivity Generation 5 Cardiac Troponin $\mathrm{T}$ Sex- and Age-Specific 99th Percentiles in the CALIPER Cohort of Healthy Children and Adolescents. Clin Chem. 2019;65 (4):589-591. doi:10.1373/clinchem.2018.299156

33. Widera C, Giannitsis E, Mueller-Hennessen M, et al. Diagnostic and prognostic value of sex- and age-specific cutpoints for high-sensitivity Troponin $\mathrm{T}$ in non-ST-elevation acute coronary syndrome. Int J Cardiol. 2019;275:13-19. doi:10.1016/j.ijcard.2018.10.027

34. Monneret D, Gellerstedt M, Bonnefont-Rousselot D. Determination of age- and sex-specific 99th percentiles for high-sensitive troponin $\mathrm{T}$ from patients: an analytical imprecision- and partitioning-based approach. Clin Chem Lab Med. 2018;56(5):818-829. doi:10.1515/cclm-2017-0256

35. Klinkenberg LJJ, Wildi K, van der Linden N, et al. Diurnal rhythm of cardiac troponin: consequences for the diagnosis of acute myocardial infarction. Clin Chem. 2016;62(12):1602-1611. doi:10.1373/clinchem.2016.257485

36. Chaulin AM, Duplyakova PD, Duplyakov DV. Circadian rhythms of cardiac troponins: mechanisms and clinical significance. Russ J Cardiol. 2020;25(3):4061. doi:10.15829/1560-4071-2020-4061

37. Chaulin AM, Duplyakov DV. High-sensitivity cardiac troponins: circadian rhythms. Cardiovasc Ther Prev. 2021;20(1):2639. doi:10.15829/1728-8800-2021-2639

38. Chaulin AM, Duplyakov DV. Environmental factors and cardiovascular diseases. Hygiene Sanitation. 2021;100(3):223-228. doi:10.47470/0016-9900-2021-100-3-223-228

39. van der Linden N, Cornelis T, Klinkenberg LJJ, et al. Strong diurnal rhythm of troponin $\mathrm{T}$, but not troponin $\mathrm{I}$, in a patient with renal dysfunction. Int $J$ Cardiol. 2016;221:287-288. doi:10.1016/j.ijcard.2016.06.268

40. Chaulin AM, Duplyakov DV. On the potential effect of circadian rhythms of cardiac troponins on the diagnosis of acute myocardial infarction. Signa Vitae. 2021;17(3):79-84. doi:10.22514/sv.2021.050

41. Chaulin AM, Duplyakov DV. Comorbidity in chronic obstructive pulmonary disease and cardiovascular disease. Cardiovascular Therapy Prevent. 2021;20(3):2539. doi:10.15829/1728-88002021-2539

42. Sigurdardottir FD, Lyngbakken MN, Holmen OL, et al. Relative Prognostic Value of Cardiac Troponin I and C-Reactive Protein in the General Population (from the Nord-Trøndelag Health [HUNT] Study). Am J Cardiol. 2018;121(8):949-955. doi:10.1016/j. amjcard.2018.01.004

43. Clerico A, Padoan A, Zaninotto M, Passino K, Plebani M. Clinical relevance of biological variation of cardiac troponins. Clin Chem Lab Med. 2020. doi:10.1515/cclm-2020-1433

44. Postigo A, Vernooij RWM, Fernández-Avilés F, Martínez-Sellés M. Cardiac troponin and infective endocarditis prognosis: a systematic review and meta-analysis. Eur Heart $J$ Acute Cardiovasc Care. 2021;10(3):356-366. doi:10.1093/ehjacc/ zuab008

45. Weckbach LT, Curta A, Bieber S, et al. Myocardial Inflammation and Dysfunction in COVID-19-Associated Myocardial Injury. Circ Cardiovasc Imaging. 2021;14(1):e012220. doi:10.1161/ CIRCIMAGING.120.011713

46. Gommans DHF, Cramer GE, Fouraux MA, et al. Usefulness of High-Sensitivity Cardiac Troponin $\mathrm{T}$ to Predict Long-Term Outcome in Patients with Hypertrophic Cardiomyopathy. Am J Cardiol. 2021;152:120-124. doi:10.1016/j.amjcard.2021.04.040

47. Wang K, Zelnick LR, Anderson A, et al. Cardiac Biomarkers and Risk of Mortality in CKD (the CRIC Study). Kidney Int Rep. 2020;5(11):2002-2012. doi:10.1016/j.ekir.2020.08.028 
48. Chaudary N, Geraci SA. Prognostic value of cardiac-specific troponins in chronic obstructive pulmonary disease exacerbations: a systematic review. J Miss State Med Assoc. 2014;55(2):40-44.

49. Chaulin AM, Abashina OE, Duplyakov DV. Pathophysiological mechanisms of cardiotoxicity in chemotherapeutic agents. Russian Open Med J. 2020;9:e0305. doi:10.15275/rusomj.2020.0305

50. Chaulin AM, Duplyakov DV. Arrhythmogenic effects of doxorubicin. Complex Issues Cardiovascular Dis. 2020;9 (3):69-80. doi:10.17802/2306-1278-2020-9-3-69-80

51. Samaha E, Brown J, Brown F, et al. High-sensitivity cardiac troponin $\mathrm{T}$ increases after stress echocardiography. Clin Biochem. 2019;63:18-23. doi:10.1016/j.clinbiochem.2018.11.013

52. Li F, Hopkins WG, Wang X, et al. Moderators and Reference Limits of Exercise-Induced Elevation of Cardiac Troponin $\mathrm{T}$ in Athletes: a Systematic Review and Meta-Analysis. Front Physiol. 2021;12:651851. doi:10.3389/fphys.2021.651851

53. Caligiuri SP, Austria JA, Pierce GN. Alarming Prevalence of Emergency Hypertension Levels in the General Public Identified by a Hypertension Awareness Campaign. Am J Hypertens. 2017;30(3):236-239. doi:10.1093/ajh/hpw136

54. Chaulin AM, Grigorieva Y, Pavlova TV, Duplyakov DV. Diagnostic significance of complete blood count in cardiovascular patients; Samara State Medical University. Russian J Cardiol. 2020;25(12):3923. doi:10.15829/1560-4071-2020-3923

55. Lawes CM, Vander Hoorn S, Rodgers A. International Society of Hypertension. Global burden of blood-pressure-related disease, 2001. Lancet. 2008;371(9623):1513-1518. doi:10.1016/S01406736(08)60655-8

56. World Health Organization. A Global Brief on Hypertension: Silent Killer, Global Public Health Crisis. 2 ed. Geneva, Switzerland: World Health Organization; 2013. https://apps.who. int/iris/bitstream/handle/10665/79059/WHO_DCO_WHD_2013. 2_rus.pdf?sequence $=5 \&$ isAllowed=y. Accessed July 8, 2021.

57. Lindner G, Pfortmueller CA, Braun CT, Exadaktylos AK. Nonacute myocardial infarction-related causes of elevated high-sensitive troponin $\mathrm{T}$ in the emergency room: a cross-sectional analysis. Intern Emerg Med. 2014;9(3):335-339. doi:10.1007/s11739-013-1030-y

58. Long B, Long DA, Tannenbaum L, Koyfman A. An emergency medicine approach to troponin elevation due to causes other than occlusion myocardial infarction. Am J Emerg Med. 2020;38 (5):998-1006. doi:10.1016/j.ajem.2019.12.007

59. Masri W, Le Guillou E, Hamdi E, et al. Troponin elevation in other conditions than acute coronary syndromes. Ann Biol Clin (Paris). 2017;75(4):411-419. doi:10.1684/abc.2017.1262

60. Stabouli S, Kotsis V, Rizos Z, et al. Left ventricular mass in normotensive, prehypertensive and hypertensive children and adolescents. Pediatr Nephrol. 2009;24(8):1545-1551. doi:10.1007/s00467-009-1165-2

61. McEvoy JW, Chen Y, Nambi V, et al. High-Sensitivity Cardiac Troponin T and Risk of Hypertension. Circulation. 2015;132 (9):825-833. doi:10.1161/CIRCULATIONAHA.114.014364

62. de Lemos JA, Drazner MH, Omland T, et al. Association of troponin $\mathrm{T}$ detected with a highly sensitive assay and cardiac structure and mortality risk in the general population. JAMA. 2010;304(22):2503-2512. doi:10.1001/jama.2010.1768

63. Uçar H, Gür M, Kivrak A, et al. High-sensitivity cardiac troponin $\mathrm{T}$ levels in newly diagnosed hypertensive patients with different left ventricle geometry. Blood Press. 2014;23(4):240-247. doi:10.3109/08037051.2013.840429

64. Harvell B, Henrie N, Ernst AA, et al. The meaning of elevated troponin I levels: not always acute coronary syndromes. Am J Emerg Med. 2016;34(2):145-148. doi:10.1016/j.ajem.2015.09.037
65. Afonso L, Bandaru H, Rathod A, et al. Prevalence, determinants, and clinical significance of cardiac troponin-I elevation in individuals admitted for a hypertensive emergency. J Clin Hypertens. 2011;13(8):551-556. doi:10.1111/j.1751-7176.2011.00476.x

66. Papadopoulos DP, Sanidas EA, Viniou NA, et al. Cardiovascular hypertensive emergencies. Curr Hypertens Rep. 2015;17(2):5. doi:10.1007/s11906-014-0515-z

67. Whelton PK, Carey RM, Aronow WS, et al. 2017 ACC/AHA/ AAPA/ABC/ACPM/AGS/APhA/ASH/ASPC/NMA/PCNA

Guideline for the Prevention, Detection, Evaluation, and Management of High Blood Pressure in Adults: a Report of the American College of Cardiology/American Heart Association Task Force on Clinical Practice Guidelines. J Am Coll Cardiol. 2018;71(19):e127-e248. doi:10.1016/j.jacc.2017.11.006

68. Acosta G, Amro A, Aguilar R, et al. Clinical Determinants of Myocardial Injury, Detectable and Serial Troponin Levels among Patients with Hypertensive Crisis. Cureus. 2020;12(1):e6787. doi: $10.7759 /$ cureus. 6787

69. Pattanshetty DJ, Bhat PK, Aneja A, Pillai DP. Elevated troponin predicts long-term adverse cardiovascular outcomes in hypertensive crisis: a retrospective study. $J$ Hypertens. 2012;30 (12):2410-2415. doi:10.1097/HJH.0b013e3283599b4f

70. Talha AM, Torres C, Del Cid J, Khan M, Rasool W, Aijaz T. The prognostic significance of highly sensitive cardiac troponin i elevation in patients presenting with hypertensive crisis. Circulation. 2019;140:A16333.

71. Omondi A, Villablanca P, Kargoli F, et al. Prevalence and prognostic significance of cardiac troponin-T elevation in patients admitted with hypertensive crises. JACC. 2017;69:1803. doi:10.1016/S0735-1097(17)35192-6

72. Cheng W, Li B, Kajstura J, et al. Stretch-induced programmed myocyte cell death. J Clin Invest. 1995;96(5):2247-2259. doi:10.1172/JCI118280

73. Singh K, Communal C, Sawyer DB, Colucci WS. Adrenergic regulation of myocardial apoptosis. Cardiovasc Res. 2000;45 (3):713-719. doi:10.1016/s0008-6363(99)00370-3

74. Singh K, Xiao L, Remondino A, Sawyer DB, Colucci WS. Adrenergic regulation of cardiac myocyte apoptosis. J Cell Physiol. 2001;189(3):257-265. doi:10.1002/jcp.10024

75. Dalal S, Connelly B, Singh M, Singh K. NF2 signaling pathway plays a pro-apoptotic role in $\beta$-adrenergic receptor stimulated cardiac myocyte apoptosis. PLoS One. 2018;13(4):e0196626. doi:10.1371/journal.pone.0196626

76. Weil BR, Suzuki G, Young RF, Iyer V, Canty JM. Troponin Release and Reversible Left Ventricular Dysfunction After Transient Pressure Overload. J Am Coll Cardiol. 2018;71 (25):2906-2916. doi:10.1016/j.jacc.2018.04.029

77. Ruwhof C, van der Laarse A. Mechanical stress-induced cardiac hypertrophy: mechanisms and signal transduction pathways. Cardiovasc Res. 2000;47(1):23-37. doi:10.1016/s0008-6363(00) 00076-6

78. van der Wees CG, Bax WH, van der Valk EJ, van der Laarse A. Integrin stimulation induces calcium signalling in rat cardiomyocytes by a NO-dependent mechanism. Pflugers Arch. 2006;451 (4):588-595. doi:10.1007/s00424-005-1402-x

79. Kandasamy AD, Chow AK, Ali MA, Schulz R. Matrix metalloproteinase-2 and myocardial oxidative stress injury: beyond the matrix. Cardiovasc Res. 2010;85(3):413-423. doi:10.1093/cvr/cvp268

80. Wang W, Schulze CJ, Suarez-Pinzon WL, Dyck JR, Sawicki G, Schulz R. Intracellular action of matrix metalloproteinase-2 accounts for acute myocardial ischemia and reperfusion injury. Circulation. 2002;106(12):1543-1549. doi:10.1161/01.cir.0000028818.33488.7b 
81. Feng J, Schaus BJ, Fallavollita JA, Lee TC, Canty JM. Preload induces troponin I degradation independently of myocardial ischemia. Circulation. 2001;103(16):2035-2037. doi:10.1161/01. cir.103.16.2035

82. Maekawa A, Lee JK, Nagaya T, et al. Overexpression of calpastatin by gene transfer prevents troponin I degradation and ameliorates contractile dysfunction in rat hearts subjected to ischemia/ reperfusion. J Mol Cell Cardiol. 2003;35(10):1277-1284. doi:10.1016/s0022-2828(03)00238-4

83. Katrukha IA, Kogan AE, Vylegzhanina AV, et al. Thrombin-Mediated Degradation of Human Cardiac Troponin T. Clin Chem. 2017;63 (6):1094-1100. doi:10.1373/clinchem.2016.266635

84. van den Born BJ, Löwenberg EC, van der Hoeven NV, et al. Endothelial dysfunction, platelet activation, thrombogenesis and fibrinolysis in patients with hypertensive crisis. $J$ Hypertens. 2011;29(5):922-927. doi:10.1097/HJH.0b013e328345023d

85. Derhaschnig U, Testori C, Riedmueller E, Aschauer S, Wolzt M, Jilma B. Hypertensive emergencies are associated with elevated markers of inflammation, coagulation, platelet activation and fibrinolysis. J Hum Hypertens. 2013;27(6):368-373. doi:10.1038/jhh.2012.53

86. Bleier J, Vorderwinkler KP, Falkensammer J, et al. Different intracellular compartmentations of cardiac troponins and myosin heavy chains: a causal connection to their different early release after myocardial damage. Clin Chem. 1998;44(9):1912-1918. doi:10.1093/clinchem/44.9.1912

87. Lazzarino AI, Hamer M, Gaze D, Collinson P, Steptoe A. The association between cortisol response to mental stress and high sensitivity cardiac troponin $\mathrm{T}$ plasma concentration in healthy adults. J Am Coll Cardiol. 2013;62(18):1694-1701. doi:10.1016/ j.jacc.2013.05.070

88. Richardson AJ, Leckie T, Watkins ER, et al. Post marathon cardiac troponin $\mathrm{T}$ is associated with relative exercise intensity. $J$ Sci Med Sport. 2018;21(9):880-884. doi:10.1016/j.jsams.2018.02.005

89. Hessel MH, Atsma DE, van der Valk EJ, Bax WH, Schalij MJ, van der Laarse A. Release of cardiac troponin I from viable cardiomyocytes is mediated by integrin stimulation. Pflugers Arch. 2008;455(6):979-986. doi:10.1007/s00424-007-0354-8

90. deFilippi C, Seliger SL, Kelley W, et al. Interpreting cardiac troponin results from high-sensitivity assays in chronic kidney disease without acute coronary syndrome. Clin Chem. 2012;58 (9):1342-1351. doi:10.1373/clinchem.2012.185322

91. Diris JH, Hackeng CM, Kooman JP, Pinto YM, Hermens WT, van Dieijen-visser MP. Impaired renal clearance explains elevated troponin $\mathrm{T}$ fragments in hemodialysis patients. Circulation. 2004;109(1):23-25. doi:10.1161/01.CIR.0000109483.45211.8F

92. Di Lullo L, Barbera V, Santoboni A, et al. Malattia renale cronica e sindrome coronarica acuta: il ruolo della troponina [Troponins and chronic kidney disease]. G Ital Nefrol. 2015;32(4):254.

93. Dubin RF, Li Y, He J; CRIC Study Investigators, et al. Predictors of high sensitivity cardiac troponin $\mathrm{T}$ in chronic kidney disease patients: a cross-sectional study in the chronic renal insufficiency cohort (CRIC). BMC Nephrol. 2013;14:229. doi:10.1186/14712369-14-229

94. Ziebig R, Lun A, Hocher B, et al. Renal elimination of troponin T and troponin I. Clin Chem. 2003;49(7):1191-1193. doi:10.1373/ 49.7.1191

95. Maruta T, Li T, Morrissey J, et al. 252: urinary Cardiac Troponin $\mathrm{I}$ is Detectable in Patients with Myocardial Injury Using a High-Sensitive Immunoassay. Crit Care Med. 2012;40:1-328. doi:10.1097/01.ccm.0000424470.26633.42

96. Pervan P, Svagusa T, Prkacin I, Savuk A, Bakos M, Perkov S. Urine high sensitive Troponin I measuring in patients with hypertension. Signa Vitae. 2017;13:62-64. doi:10.22514/ SV133.062017.13
97. Muslimovic A, Fridén V, Tenstad O, et al. The Liver and Kidneys mediate clearance of cardiac troponin in the rat. Sci Rep. 2020;10 (1):6791. doi:10.1038/s41598-020-63744-8

98. Kostyukevich OI. Arterial hypertension and liver diseases: in search of a compromise. Russian Med J. 2011;19(5):338-342.

99. Shah AS, Chin CW, Vassiliou V, et al. Left ventricular hypertrophy with strain and aortic stenosis. Circulation. 2014;130 (18):1607-1616. doi:10.1161/CIRCULATIONAHA.114.011085

100. Shah AS, Griffiths M, Lee KK, et al. High sensitivity cardiac troponin and the under-diagnosis of myocardial infarction in women: prospective cohort study. BMJ. 2015;350:g7873. doi:10.1136/bmj.g7873

101. Gore MO, Seliger SL, Defilippi CR, et al. Age- and sex-dependent upper reference limits for the high-sensitivity cardiac troponin $\mathrm{T}$ assay. $J$ Am Coll Cardiol. 2014;63 (14):1441-1448. doi:10.1016/j.jacc.2013.12.032

102. High-Sensitivity Cardiac Troponin I and T Assay Analytical Characteristics Designated by Manufacturer IFCC Committee on Clinical Applications of Cardiac Bio-Markers (C-CB). Available from: https://www.ifcc.org/media/478231/high-sensitivity-cardiac -troponin-i-and-t-assay-analytical-characteristics-designated-bymanufacturer-v122019.pdf. Accessed July 8, 2021.

103. Morrow DA, Cannon CP, Jesse RL, et al.; National Academy of Clinical Biochemistry. National Academy of Clinical Biochemistry Laboratory Medicine Practice Guidelines: clinical characteristics and utilization of biochemical markers in acute coronary syndromes. Circulation. 2007;115(13):e356-75. doi:10.1161/CIRCULATIONAHA.107.182882

104. Chaulin AM, Abashina OE, Duplyakov DV. High-sensitivity cardiac troponins: detection and central analytical characteristics. Cardiovascular Therapy Prevent. 2021;20(2):2590. doi:10.15829/1728-8800-2021-2590

105. Jeremias A, Gibson CM. Narrative review: alternative causes for elevated cardiac troponin levels when acute coronary syndromes are excluded. Ann Intern Med. 2005;142(9):786-791. doi:10.7326/0003-4819-142-9-200505030-00015

106. Horwich TB, Patel J, MacLellan WR, Fonarow GC. Cardiac troponin I is associated with impaired hemodynamics, progressive left ventricular dysfunction, and increased mortality rates in advanced heart failure. Circulation. 2003;108(7):833-838. doi:10.1161/01.CIR.0000084543.79097.34

107. Chaulin AM, Duplyakova PD, Bikbaeva GR, Tukhbatova AA, Grigorieva EV, Duplyakov DV. Concentration of high-sensitivity cardiac troponin I in the oral fluid in patients with acute myocardial infarction: a pilot study. Russian $J$ Cardiol. 2020;25 (12):3814. doi:10.15829/1560-4071-2020-3814

108. Chaulin AM, Karslyan LS, Bazyuk EV, Nurbaltaeva DA, Duplyakov DV. Clinical and Diagnostic Value of Cardiac Markers in Human Biological Fluids. Kardiologiia. 2019;59 (11):66-75. doi:10.18087/cardio.2019.11.n414

109. Chaulin AM, Duplyakov DV. Increased natriuretic peptides not associated with heart failure. Russian J Cardiol. 2020;25:4140. doi:10.15829/1560-4071-2020-4140

110. Joharimoghadam A, Tajdini M, Bozorgi A. Salivary B-type natriuretic peptide: a new method for heart failure diagnosis and follow-up. Kardiol Pol. 2017;75(1):71-77. doi:10.5603/KP. a2016.0097

111. Chen JY, Lee SY, Li YH, Lin CY, Shieh MD, Ciou DS. Urine High-Sensitivity Troponin I Predict Incident Cardiovascular Events in Patients with Diabetes Mellitus. J Clin Med. 2020;9 (12):3917. doi:10.3390/jcm9123917

112. Potkonjak AM, Sabolović Rudman S, Nikolac Gabaj N, et al. Urinary troponin concentration as a marker of cardiac damage in pregnancies complicated with preeclampsia. Med Hypotheses. 2020;144:110252. doi:10.1016/j.mehy.2020.110252 


\section{Publish your work in this journal}

Vascular Health and Risk Management is an international, peerreviewed journal of therapeutics and risk management, focusing on concise rapid reporting of clinical studies on the processes involved in the maintenance of vascular health; the monitoring, prevention and treatment of vascular disease and its sequelae; and the involvement of metabolic disorders, particularly diabetes. This journal is indexed on PubMed Central and MedLine. The manuscript management system is completely online and includes a very quick and fair peerreview system, which is all easy to use. Visit http://www.dovepress. com/testimonials.php to read real quotes from published authors. 